\title{
Predicting Autism Spectrum Disorder in Young Children: The Predictive Utility of the Ghuman-Folstein Screen for Social Interaction and Sociodemographic Factors
}

\author{
Dana K. Princiotta ${ }^{1}$, Sarah Hamill Skoch ${ }^{2 *}$, Randi Phelps ${ }^{3}$, Richard J. Morris ${ }^{1}$, \\ Nicholas Breitborde ${ }^{4}$, Irena Bukeliss ${ }^{5}$, Marco Grados ${ }^{6}$, Jaswinder K. Ghuman? \\ ${ }^{1}$ University of Arizona, Tucson, USA \\ ${ }^{2}$ College of Medicine, University of Cincinnati, Cincinnati, USA \\ ${ }^{3}$ Miami University, Oxford, USA \\ ${ }^{4}$ Ohio State University Wexner Medical Center, Columbus, USA \\ ${ }^{5}$ University of Alabama at Birmingham, Birmingham, USA \\ ${ }^{6}$ School of Medicine, Johns Hopkins University, Baltimore, USA \\ ${ }^{7}$ Medical School, University of Pittsburgh, Pittsburgh, USA \\ Email: ^hamillsk@ucmail.uc.edu
}

How to cite this paper: Princiotta, D. K., Skoch, S. H., Phelps, R., Morris, R. J., Breitborde, N., Bukelis, I., Grados, M., \& Ghuman, J. K. (2020). Predicting Autism Spectrum Disorder in Young Children: The Predictive Utility of the Ghuman-Folstein Screen for Social Interaction and Sociodemographic Factors. Psychology, 11, 1054-1066. https://doi.org/10.4236/psych.2020.117069

Received: February 7, 2020

Accepted: July 27, 2020

Published: July 30, 2020

Copyright $\odot 2020$ by author(s) and Scientific Research Publishing Inc. This work is licensed under the Creative Commons Attribution International License (CC BY 4.0).

http://creativecommons.org/licenses/by/4.0/ (c) (i) Open Access

\begin{abstract}
Background: Autism Spectrum Disorder (ASD) is characterized by having deficits in social interactions. Screening measures for ASD are often used for children over five years of age, ultimately leading to diagnosis later in development. Identification of ASD in young children is critical for early intervention. Aims: The purpose of the present study was to examine whether prediction of ASD could be improved in young children by combining social interaction scores, as measured by the Ghuman-Folstein Screen for Social Interaction (GF-SSI), with the presence of selected demographic variables (sex, age, ethnicity, mother's educational level, and socioeconomic status). Methods and Procedures: One-hundred and seventy-one clinically referred children with previously diagnosed ASD or non-ASD developmental disorders and their caregivers were included in the study. Caregivers completed a sociodemographic survey and the GF-SSI. Outcomes and Results: Results demonstrated that the final model correctly identified $74 \%$ of the cases, and the GF-SSI was found to be the greatest predictor of children having ASD. The selected demographic variables were not found to be significant predictors of the diagnosis of ASD. Conclusions and Implications: These results are discussed in relation to the literature on predicting ASD in young children. Future directions for research are also discussed.
\end{abstract}




\section{Keywords}

Autism Spectrum Disorder, Ghuman-Folstein Screen for Social Interaction, Screening Tool, Early Identification/Detection

\section{Introduction}

Autism Spectrum Disorder (ASD) is characterized as a combination of impairments in the areas of social interaction (e.g., poor eye contact, lack of gestures, lack of friendships, lack of empathy), communication (e.g., speech, echolalia, pronoun reversal, lack of pretend play), patterns of behavior, interests and activities (e.g., narrow interests, rigidity, and hand flapping (APA, 2013).

About 1 in 59 children are identified with an ASD (ADDMN, 2014). Early identification of ASD, or previously autistic disorder, is vital as it provides the best opportunity for early intervention, thus maximizing prospects for improved short and long-term developmental outcomes (Barbaro \& Dissanayake, 2009). As a result, mental health professionals are interested in identifying early indicators that may help to predict at an early age which children will go on to develop an ASD.

\subsection{Screening Tools}

Screening tools are of great interest to mental health professionals to assist in identifying at-risk youth. In a study comparing different screening instruments in more than 200 children, many screening measures failed to obtain the level of sensitivity and specificity needed to distinguish children with ASD from at-risk children with other developmental difficulties who did not have an ASD diagnosis (Osterling et al., 2009). In fact, Osterling and colleagues (2009) suggest that no individual test item or assessment instrument thus far has shown adequate power in discriminating children with ASD from those with a non-ASD diagnosis. Gray, Tonge, and Sweeney (2008) echo similar sentiment, reporting that many of the assessment instruments available for assessing ASD have not been validated within community healthcare settings. The lack of early childhood screening instruments for assessing ASD has, therefore, led to diagnosing ASD late in the child's development or even into young adulthood (Matson, 2007). In this regard, most childhood assessment and screening instruments for ASD are not utilized prior to two years of age and are more frequently used when a child reaches at least six years of age (Matson, 2007). Zwaigenbaum and colleagues (2015) suggested that while ASD screenings prior to two years of age may be associated with higher false positive rates, screening may provide valuable information and increase early detection.

Social interaction problems are considered a core deficit in autism spectrum disorders (ASDs) and are the single most powerful predictor for diagnosing ASDs (Volkmar, Chawarska, \& Klin, 2005; Ghuman, Leone, Lecavalier, \& Landa, 
2011). Social interaction problems manifest early in young at-risk children. Problems in joint attention, social responsiveness, pretend play skills, motor imitation, and use of conventional gestures have been identified in a number of studies in young children with ASD (Landa, Holman, \& Garrett-Mayer, 2007; Ventola et al., 2007). Impairment in joint attention is one of the most reliable markers of ASDs (Ventola et al., 2007). Joint attention typically emerges between 6 and 18 months of age (Mundy \& Burnette, 2005) and can potentially help screen young at-risk children. On the other hand, restricted interests/repetitive behaviors that are characteristic of ASDs (e.g., extreme interest in one topic, hand flapping or rocking, or repetitive verbal behaviors), may begin to emerge later than other social indicators (Volkmar et al., 2005). Furthermore, there is evidence that restricted interests/repetitive behaviors failed to differentiate ASD children from non-ASD children at 20 and at 42 months of age (Wiggins \& Robbins, 2008). Given that restricted interests/repetitive behaviors may not be early indicators of ASDs, screening efforts for early identification in young children should attend to basic social interaction behaviors (i.e., joint attention, imitation, gestures, and play behaviors) that seem to distinguish ASDs from other non-ASD developmental disorders in early toddlerhood.

\subsection{Ghuman-Folstein Screen for Social Interaction}

The Ghuman-Folstein Screen for Social Interaction (GF-SSI) offers a way to specifically screen social interaction deficits via parent-caregiver report (Ghuman, Leone, Lecavalier, \& Landa, 2011). The GF-SSI is a screening instrument developed to examine a child's reciprocal social interaction and joint attention skills as such problems are considered core deficits in ASDs and manifest early in development in at-risk children (Ghuman et al., 2011; Ventola et al., 2007). Ghuman and colleagues (2011) found that the GF-SSI reliably discriminates children diagnosed with ASDs from children with other developmental and/or psychiatric disorders and can be helpful in screening young at-risk children in clinical high-risk populations. Diagnostic validity was found to be equally strong for both matched and unmatched samples of younger (24 - 42 months old) and older (43 - 61 months old) preschool-age children (Ghuman et al., 2011). High sensitivity and specificity were obtained with the screening cutoff scores established through ROC analysis (Ghuman et al., 2011). Scoring recommendations were provided based on stringent diagnostic comparisons between overall ASD (autism and Pervasive Developmental Disorder Not Otherwise Specified (PDD-NOS) combined) and non-ASD clinical referrals and incorporated the relative benefits of both the greater experimental control of the matched and the greater statistical power of the larger unmatched samples (Ghuman et al., 2011).

\subsection{Sociodemographic Factors}

In addition to screening tools, an increasing number of studies point to several sociodemographic factors (e.g., gender, age, ethnicity, mother's level of educa- 
tion, socioeconomic status) that may be associated with ASD (e.g., Hertz-Picciotto et al., 2006). For example, males have consistently been shown to have a higher risk of ASD than females (4:1; Centers for Disease Control and Prevention, 2009; Croen, Grether, \& Selvin, 2002). Additionally, age of diagnosis is a strong prognostic predictor for early intervention, which in turn can reduce the need for more intensive special education services at later ages (Harris \& Handleman, 2000) and improve prognosis (Matson \& Minshawi, 2006). Other sociodemographic variables such as ethnicity, mother's level of education and socioeconomic status (SES), parental age, and birth order have been inconsistent predictors of an ASD (Bhasin \& Schendel, 2007; Croen, Grether, \& Selvin, 2002; Mandell et al., 2002; Feldman et al., 2015).

\subsection{The Present Study}

Given the limitations in previous research, the first aim of the present study was to examine the utility of social interaction in differentiating between ASD and developmental and psychiatric disorders in a heterogeneous high-risk clinical sample of children between 24 and 60 months of age. Due to discrepancies in findings across the literature, the second aim examined whether selected theoretically-derived sociodemographic variables (i.e., ethnicity, mother's education level, and socioeconomic status; e.g., Hertz-Picciotto et al., 2006; Mandell et al., 2002; Matson, 2007; Charlop-Christy et al., 2008; Matson \& Minshawi, 2006) could further contribute to this diagnostic differentiation.

Based on previous research (e.g., Ghuman et al., 1998a, 1998b, 2011; Osterling et al., 2009), we hypothesized that social interaction deficits as measured by the GF-SSI would significantly predict $(p<0.05)$ the probability of identifying children with a diagnosis of ASDs in a sample of clinically referred high-risk children between 24 and 60 months-of-age who have previously been diagnosed as having either an ASDs or another developmental and/or psychiatric disorder. Second, we hypothesized that the GF-SSI score would be the best predictor of an ASDs diagnosis in the final probability prediction model for those children having an ASDs and that the GF-SSI score and child's sex (i.e., male gender) will significantly predict ASDs in the present sample. Third, we conducted an exploratory analysis of the sociodemographic variables of ethnicity, mother's education level, and SES to examine if these variables were associated with an ASDs diagnosis.

\section{Methods}

\subsection{Study Population}

All study procedures were in accordance with the University of Arizona Human Subjects Protection Program. This study used a subset of children recruited for a larger study of children with Autism Spectrum and developmental disorders (Ghuman, Leone, Lecavalier, \& Landa, 2011). Participants were 171 clinically-referred children recruited during clinic appointments in various autism, 
psychiatry, developmental, or specialty research clinics for genetics and developmental disorders (59 children with diagnosis of ASD, 44 children with diagnosis of PDD-NOS and 68 children diagnosed with other non-ASD developmental and/or psychiatric disorder). The group of the 68 children with other non-ASD developmental and/or psychiatric disorders is hereafter referred as Non-ASD Clinical Diagnosis Group; these children were diagnosed with one of the following Diagnostic and Statistical Manual of Mental Disorders (DSM) IV diagnoses: Communication Disorder, Fragile X Syndrome, Attention Deficit/ Hyperactivity Disorder, Oppositional Defiant Disorder, Separation Anxiety Disorder, Generalized Anxiety Disorder, or Depressive Disorder, and Mood Disorder. In the most recent version of the DSM (DSM-V), ASD is now inclusive of autistic disorder, Asperger's disorder, pervasive developmental disorder not otherwise specified (PDD-NOS) and childhood disintegrative disorder, viewing these conditions as existing on a continuum rather than categorical. For the present study, DSM-IV-R (APA, 2000) diagnoses of ASD were used and were based on an expert clinical assessment conducted by a professional or a multidisciplinary team and supported by the Autism Diagnostic Interview-Revised (ADI-R; Lord et al., 2000), Childhood Autism Rating Scale (CARS) (Schopler, Reichler, \& Renner, 1986), or both DSM-IV-R (APA, 2000).

\subsection{Measures}

\subsubsection{Social Interaction Capacity}

The Ghuman-Folstein Screen for Social Interaction (GF-SSI; Ghuman, Freund, Reiss, Serwint, \& Folstein, 1998a) is a parent/caregiver questionnaire designed to measure social interactions in young children (24 - 61 months). Fifty-four items based on the ADI-R, Vineland Adaptive Behavior Scales (Rutter, LeCouteur, \& Lord, 2003) and clinical expertise measure a child's reciprocal social interaction capacity taking into account the child's cognitive level (Ghuman et al., 2011). In contrast to other screening tools, the GF-SSI focuses on typical social interaction development to screen at-risk children for ASDs, higher scores on the GF-SSI are indicative of more normative social interaction capacity and lower scores indicate impaired or delayed social interaction development. Moderate convergence with ASD diagnostic measures was demonstrated to significantly discriminate ASD and non-ASD clinical groups. High sensitivity and specificity values were obtained for discriminating ASD and non-ASD clinical participants and screening cut-off scores were established based on ROC analysis (Ghuman et al., 2011).

\subsubsection{Sociodemographic Factors}

Other predictive variables that were coded and examined included: age of child (in months), sex, ethnicity, maternal education and socioeconomic status (SES). Socioeconomic status was determined using the Hollingshead Four Factor Index of Social Status (Hollingshead, 1975).

Diagnosis of $A S D$ or non- $A S D$ 
The outcome measure for this investigation was the diagnosis given to the child: 1) ASD, 2) PDD-NOS or 3) non-ASD clinical diagnosis as described in the study population.

\subsection{Data Analyses}

Data analyses were completed using the SPSS for Windows statistical package 18.0 PASW statistics version 18.0.0 for Windows (IBM Corporation, Somers, NY, USA). Descriptive analyses were conducted first to compare the dependent variable with each independent variable. Bivariate analyses were computed using phi correlations between the dependent variable and dichotomous independent variables. Spearman's rank correlations were computed between the dependent variable and continuous independent variables. Binary logistic regression was used to predict the probability of ASD by fitting the logistic model to the data (McCulloch, 2000). Logistic regression is a commonly used technique for modeling a binary response variable as a function of predictor variables (McCulloch, 2000). For the binary logistic regression conducted to test the predictive ability of social interaction deficits in predicting a diagnosis of ASD versus PDD-NOS and other non-ASD developmental and/or psychiatric disorders, children were grouped into two categories: 1) ASD and 2) a Combined Clinical Category consisting of 112 children (44 children with PDD-NOS and 68 children with other non-ASD developmental and/or psychiatric disorder).

\section{Theory/Calculation}

Autism Spectrum Disorders often go misdiagnosed or undiagnosed until middle to late childhood which consequently delays socioemotional and academic intervention. While comprehensive assessments (e.g., Autism Diagnostic Observation Schedule) are considered the gold standard for diagnosing ASDs, screening tools (i.e., GF-SSI) are critical for early detection of toddlers and young children who experience symptoms related to ASDs. Brief screening measures sensitive enough to detect atypical development in toddlerhood or early childhood will allow for individuals in early developmental periods to be identified to seek further comprehensive evaluation. The GF-SSI is a useful tool for identifying individuals with ASDs; however, it is important to determine whether the GF-SSI can accurately detect ASDs in children between the ages of 20 and 60 months. Additionally, sociodemographic factors may account for unique variance in the relation between GF-SSI and ASDs, and it is unknown if the GF-SSI is sensitive enough to significantly predict ASDs above and beyond sociodemographic factors.

\section{Results}

\subsection{Descriptive Statistics}

Descriptive data for children with ASD $(N=59)$, PDD-NOS $(N=44)$, and non-ASD clinical Diagnoses $(N=68)$ are listed in Table 1 . There were several 
Table 1. Descriptive statistics.

\begin{tabular}{|c|c|c|c|}
\hline & $\begin{array}{l}\text { ASD Group } \\
(\mathrm{N}=59)\end{array}$ & $\begin{array}{l}\text { PDD-NOS Group } \\
\qquad(\mathrm{N}=44)\end{array}$ & $\begin{array}{l}\text { Non-ASD Clinical } \\
\text { Diagnosis Group } \\
\quad(\mathrm{N}=68)\end{array}$ \\
\hline Age (years) & $M=3.63 ; S D=0.74^{\mathrm{a}}$ & $M=3.58 ; S D=0.81^{\mathrm{b}}$ & $M=3.92 ; S D=0.75^{\mathrm{ab}}$ \\
\hline African-American (\%) & $17 \%^{\mathrm{c}}$ & $10 \%^{\mathrm{d}}$ & $35 \%$ cd \\
\hline Asian-American (\%) & $2 \%$ & $2 \%$ & $0 \%$ \\
\hline Caucasian $(\%)$ & $76 \%$ & $83 \%{ }^{\mathrm{e}}$ & $62 \%^{\mathrm{e}}$ \\
\hline Hispanic (\%) & $0 \%$ & $2 \%$ & $3 \%$ \\
\hline Native American (\%) & $2 \%$ & $2 \%$ & $0 \%$ \\
\hline Other & $3 \%$ & $0 \%$ & $0 \%$ \\
\hline Maternal Education (\% with BA) & $55 \%$ & $46 \%$ & $43 \%^{¥}$ \\
\hline SES & $M=33.64 ; S D=20.22^{* \mathrm{f}}$ & $M=31.05 ; S D=19.66^{\dagger g}$ & $M=43.66 ; S D=23.20 \ddagger^{\mathrm{fg}}$ \\
\hline Sex (\% male) & $81 \%$ & $82 \%$ & $71 \%$ \\
\hline SSI & $M=70.54 ; S D=26.18^{\mathrm{hi}}$ & $M=83.75 ; S D=24.86^{\mathrm{ij}}$ & $M=106.09 ; S D=24.86^{\mathrm{hj}}$ \\
\hline
\end{tabular}

Note. Superscript letters indicate pairs were $p<0.05$. Percentages for race/ethnicity do not always equal $100 \%$ due to rounding. ${ }^{*} N=55,{ }^{\dagger} N=41, \ddagger N=56$, $¥ N=61$. 
ing Caucasian), maternal education, and socioeconomic status, social interaction deficits significantly predicted the ASD Group while social interaction deficits did not significantly predict Combined Clinical categories (children in the PDD NOS and Non-ASD Clinical Diagnosis groups) among the entire sample (Wald $=23.28 ; p<0.001 ; O R=0.96)$. More specifically, lower scores on the GF-SSI predicted increased association with an ASD diagnosis, meaning that the children with decreased social interaction scores were related to an increased likelihood of having an ASD diagnosis. Subsequent follow-up analyses controlling for the same demographic variables demonstrated that the GF-SSI scores accurately differentiated between: 1) children with an ASD versus children with PDD-NOS ( Wald $=6.00 ; p=0.01 ; O R=0.98) ; 2$ ) children with an ASD versus children without an ASD diagnosis, the Combined Clinical category (Wald $=22.84 ; p<$ $0.001 ; O R=0.94)$; and 3 ) children with PDD-NOS versus children with non-ASD developmental and/or psychiatric disorders, the Clinical Diagnosis Group ( Wald $=8.39 ; p=0.004 ; O R=0.96$ ). Thus, the GF-SSI tool was meaningful in distinguishing children with or without an ASD diagnosis.

\section{Discussion}

The purpose of the present study was to examine whether an Autism Spectrum Disorder diagnosis could be predicted on the basis of a child's social interaction, as measured by the GF-SSI and theoretically driven demographic variables (e.g., sex, age, ethnicity, mother's educational level, and socioeconomic status; Mandell et al., 2009; Matson \& Minshawi, 2006; Bhasin \& Schendel, 2007; Croen, Grether, and Selvin, 2002) between the ages of 24 and 60 months. As hypothesized, the results demonstrated that an ASD diagnosis was significantly predicted, on the basis of children's social interaction scores as measured by the GF-SSI. Specifically, the GF-SSI score aided in the prediction of a diagnosis of ASD when it was added in the final step of the model for Parts 1 (ASD versus all other clinical diagnoses), 2 (ASD diagnosis versus PDD-NOS diagnosis), and 3 (ASD diagnosis versus Non-ASD clinical diagnoses) of these analyses. The final model was able to correctly identify a higher percentage of the diagnosed cases from the null model. The GF-SSI scores of children were also found to be negatively correlated with a diagnosis of ASD as discovered in the bivariate analyses of Part 1 (ASD versus all other clinical diagnoses), meaning that increased scores on the GF-SSI (i.e., higher scores = increased social interaction) were correlated with decreased likelihood of an ASD diagnosis. Children with more social interaction ability were less likely to have an ASD diagnosis. The model was not found to be significant until GF-SSI scores were added during the final step of the model building process.

GF-SSI was found to be a significant predictor of an ASD diagnosis even after controlling for sociodemographic variables. In this regard, a strong bivariate relationship was found between GF-SSI and ASD prior to the binary logistic regression analyses. As few screening measures currently exist with the level of 
sensitivity and specificity needed to distinguish children with ASD from children with abnormal development (Osterling et al., 2009), the GF-SSI is indicated to be a strong predictor for ASD and may be a useful screening tool to discriminate between children with ASD versus children with PDD-NOS and other clinical diagnoses ages 2 to 6 years old.

The predictors of sex, age, ethnicity, mother's educational level, and socioeconomic status were not significant predictors of the diagnosis of ASD. Although many studies have reported a 4:1 ratio for males versus females in the general pediatric population with regard to the diagnosis of ASD (e.g., Banach et al., 2009; Centers for Disease Control and Prevention, 2009; Charlop-Christy et al., 2008; Croen, Grether, \& Selvin, 2002), we did not replicate those findings in our sample. We did not find age to be a significant predictor of a diagnosis of ASD in children $2-5$ years of age. Our model is consistent with the literature that most children are not diagnosed until $41 / 2$ to $51 / 2$ years of age (e.g., school age children; citation).

Regarding ethnicity, while a large body of research points to ethnic disparities in the diagnosis of mental health disorders in children generally (Mandell et al., 2009), the evidence is less conclusive for ASD, as was also demonstrated in the current study. The majority of the children in our sample were Caucasian, followed by African American, and, contrary to other findings (Mandell et al., 2009), there was not a significant difference by ethnicity in the diagnosis of ASD. Likewise, the findings in the present study do not support maternal education as a significant predictor of ASD. Although Bhasin and Schendel (2007) found in previous research that higher family income was significantly associated with ASD, consistent with the present findings, SES has not, in general, been found to predict ASD (e.g., Begeer et al., 2009; Croen, Grether, \& Selvin, 2002). The current study highlights the importance of considering sociodemographic factors in the prediction of ASDs. Although additional research needs to be done in order to determine the role of sociodemographic factors in different samples to increase generalizability, this study suggests that sociodemographic variables should not be relied upon when predicting risk for ASD but should be considered as possible covariates in analyses. Moreover, the current study suggests that the GF-SSI is sensitive enough to detect subtle distinctions between individuals with ASDs and those without ASDs.

\subsection{Limitations of the Present Study}

The results of the present study are best understood within the context of its limitations. Due to the clinical and heterogeneous nature of the sample, a standard assessment battery was not administered across participants. As recommended by Charman and Baird (2002), ASD diagnoses were determined via detailed clinical assessment by experienced professionals in all cases and were aided by the ADI-R and/or the CARS in most cases. Second, this study examined children between the ages of 24 and 60 months who were diagnosed as having an 
ASD diagnosis, PDD-NOS, or a non-ASD developmental and/or psychiatric diagnosis. Much of the research focuses on children that span the age of $4-10$ years of age, rather than the $2-5$ age range in the present study. This truncation of the dataset leads to loss in sample size and limits our ability to generalize these findings to older children. Additionally, the number of children included in this study was small, limiting our power and effect sizes.

While age restriction is a limitation of the current study, it is also a primary strength. One goal of the current study was to determine the possible predictive utility of the GF-SSI to identify children at-risk for ASDs in early development periods. Indeed, these results demonstrate that sensitivity and flexibility of the GF-SSI such that this measure may be used in early ages to identify individuals at-risk. Importantly, however, the current study relied upon a clinical sample of individuals who had previously been diagnosed with ASDs or a non-ASDs developmental disorder. This limits our ability to suggest that this measure may be sensitive enough to be an early screening tool for individuals that have not yet been diagnosed. Given the demonstrated sensitivity to differences in presentation, future longitudinal work is needed to adequately determine the early predictive utility of this measure prior to diagnosis.

Despite these limitations, the present study highlights the need to continue studying the demographic factors associated with a diagnosis of ASD. Although significant results were found for the GF-SSI, future studies examining larger samples of children with ASD diagnoses would enhance predictive power of the variables examined in this study.

\subsection{Conclusion}

The present study supports the discriminative ability of the GF-SSI as a screening tool in clinical settings to distinguish an ASD diagnosis from a non-ASD diagnosis above and beyond standard clinical instruments (i.e., Autism Diagnostic Interview-Revised and the Childhood Autism Rating Scales). This study was conducted in a heterogeneous clinic sample of children with a variety of delays and abnormalities. This likely increases the generalizability of the present findings and adds to the potential strength of using the GF-SSI as a clinical tool.

Early identification of children with ASD is crucial in providing children and families with early intervention. The identification of social interaction and joint attention deficits is beneficial in that this type of assessment can guide treatment recommendations for parents. Families may be encouraged to promote social interaction, increase eye contact, and use scaffolding techniques with their child at an early age. Given that few reliable and valid screening instruments exist for young children between two and four years of age, this screening measure offers clinicians a tool to use to assist in the early identification of ASD among young at-risk children.

\section{Acknowledgements}

We would like to thank and acknowledge the families who participated in this 
research. Additionally, this article is dedicated to the work and memory of Dr. Jaswinder Ghuman. Dr. Ghuman was born in Dakha, India, attended C.M.C Medical College in Ludhiana, Punjab, India and also received Internal Medicine training at C.M.C. She then moved to America with her husband, Dr. Hari Ghuman, and received psychiatric training at Rochester Psychiatric Center, Washington University in St. Louis and Johns Hopkins Medical School in Baltimore. She held faculty positions in child psychiatry at Johns Hopkins Medical School, University of Arizona Medical College, and University of Pittsburgh Medical School. Dr. Ghuman received several teaching awards for her training of a number of child psychiatrists, general psychiatry residents, psychology interns and medical students. As a child psychiatrist, Dr. Ghuman invested her life in caring for infants and preschool children with mental health and developmental issues. She was crucial in the design, oversight, and written components of this manuscript and this project would not have been possible without her. We dedicate this article to Dr. Ghuman's memory, her impact on the children and families she touched throughout her career, and to the lasting impact she left on her colleagues.

\section{Conflicts of Interest}

The authors declare no conflicts of interest regarding the publication of this paper.

\section{References}

ADDMN. Autism and Developmental Disabilities Monitoring Network Surveillance Year 2010 Principal Investigators (2014). Prevalence of Autism Spectrum Disorder among Children Aged 8 Years-Autism and Developmental Disabilities Monitoring Network, 11 Sites, United States, 2010. Morbidity and Mortality Weekly Report: Surveillance Summaries, 63, 1-21.

APA (American Psychiatric Association) (2000). Diagnostic and Statistical Manual for Mental Disorders (4th ed., Text Revision). Washington DC: American Psychiatric Association.

APA (American Psychiatric Association) (2013). Diagnostic and Statistical Manual of Mental Disorders (5th ed.). Washington DC: American Psychiatric Association. https://doi.org/10.1176/appi.books.9780890425596

Banach, R., Thompson, A., Szatmari, P., Goldberg, J., Tuff, L., Zwaigenbaum, L., \& Mahoney, W. (2009). Brief Report: Relationship between Non-Verbal IQ and Gender in Autism. Journal of Autism and Developmental Disorders, 39, 188-193. https://doi.org/10.1007/s10803-008-0612-4

Barbaro, J., \& Dissanayake, C. (2009). Autism Spectrum Disorders in Infancy and Toddlerhood: A Review of the Evidence on Early Signs, Early Identification Tools, and Early Diagnosis. Journal of Developmental \& Behavioral Pediatrics, 30, 447-459. https://doi.org/10.1097/DBP.0b013e3181ba0f9f

Begeer, S., El Bouk, S., Boussaid, W., Terwogt, M. M., \& Koot, H. M. (2009). Underdiagnosis and Referral Bias of Autism Ethnic Minorities. Journal of Autism and Developmental Disorders, 39, 142-148. https://doi.org/10.1007/s10803-008-0611-5

Bhasin, T. K., \& Schendel, D. (2007). Sociodemographic Risk Factors for Autism in a US 
Metropolitan Area. Journal of Autism and Developmental Disorders, 37, 667-677. https://doi.org/10.1007/s10803-006-0194-y

Centers for Disease Control and Prevention (2009). Autism and Family History. https://www.cdc.gov/ncbddd/autism/addm-community-report/index.html

Charlop-Christy, M., Schreibman, L., Berry, D., \& Rocha, M. (2008). Childhood Autism, In R. Morris, \& T. Kratochwill (Eds.), The Practice of Child Therapy (4th ed.). Boston, MA: Allyn \& Bacon.

Charman, T., \& Baird, G. (2002). Practitioner Review: Diagnosis of Autism Spectrum Disorder in 2- and 3-Year-Old Children. Journal of Child Psychology and Psychiatry, 43, 289-305. https://doi.org/10.1111/1469-7610.00022

Croen, L. A., Grether, J. K., \& Selvin, S. (2002). Descriptive Epidemiology of Autism in a California Population: Who Is at Risk? Journal of Autism and Developmental Disorder, 32, 217-224. https://doi.org/10.1023/A:1015405914950

Feldman, M. A., Hendry, A. M., Ward, R. A., Hudson, M., \& Liu, X. (2015). Behavioral Development and Sociodemographics of Infants and Young Children at Higher and Lower Risk for Autism Spectrum Disorders. Journal of Autism and Developmental Disorders, 45, 1167-1175. https://doi.org/10.1007/s10803-014-2277-5

Ghuman, J. K., Freund, L., Reiss, A., Serwint, J., \& Folstein, S. (1998a). Early Detection of Social Interaction Problems: Development of a Social Interaction Instrument in Young Children. Journal of Developmental and Behavioral Pediatrics, 19, 411-419. https://doi.org/10.1097/00004703-199812000-00004

Ghuman, J. K., Peebles, C. D., \& Ghuman, H. S. (1998b). Review of Social Interaction Measures in Infants and Preschool Children. Infants and Young Children, 11, 21-44. https://doi.org/10.1097/00001163-199810000-00006

Ghuman, J. K., Leone, S., Lecavalier, L., \& Landa, R. (2011). The Screen for Social Interaction (SSI): A Screening Measure for Autism Spectrum Disorders in Preschoolers. Research in Developmental Disabilities, 32, 2519-2529. https://doi.org/10.1016/j.ridd.2011.07.008

Gray, K. M., Tonge, B. J., \& Sweeney, D. J. (2008). Screening for Autism in Young Children with Developmental Delay: An Evaluation of the Developmental Behaviour Checklist: Early Screen. Journal of Autism and Developmental Disorders, 38, 1003-1010. https://doi.org/10.1007/s10803-007-0473-2

Harris, S. L., \& Handleman, J. S. (2000). Age and IQ at Intake as Predictors of Placement for Young Children with Autism: A Four- to Six-Year Follow-Up. Journal of Autism and Developmental Disorders, 30, 137-142. https://doi.org/10.1023/A:1005459606120

Hertz-Picciotto, I., Croen, L. A., Hansen, R., Jones, C. R., Van de Water, J., \& Pessah, I. N. (2006). The CHARGE Study: An Epidemiologic Investigation of Genetic and Environmental Factors Contributing to Autism. Environmental Health Perspectives, 114, 1119-1125. https://doi.org/10.1289/ehp.8483

Hollingshead, A. B. (1975). Four Factor Index of Social Status. New Haven, CT: Department of Sociology, Yale University.

Landa, R. J., Holman, K. C., \& Garrett-Mayer, E. (2007). Social and Communication Development in Toddlers with Early and Later Diagnosis of Autism Spectrum Disorders. Archives of General Psychiatry, 64, 853-864. https://doi.org/10.1001/archpsyc.64.7.853

Lord, C., Risi, S., Lambrecht, L., Cook, E. H., Leventhal, B. L., DiLavore, P. C., Pickles, A., \& Rutter, M. (2000). The Autism Diagnostic Observation Schedule-Generic: A Standard Measure of Social and Communication Deficits Associated with the Spectrum of Autism. Journal of Autism and Developmental Disorders, 30, 205-223. https://doi.org/10.1023/A:1005592401947 
Mandell, D. S., Listerud, J., Levy, S. E., \& Pinto-Martin, J. A. (2002). Race Differences in the Age at Diagnosis among Medicaid-Eligible Children with Autism. Journal of American Academic Child Adolescent Psychiatry, 41, 1447-1453. https://doi.org/10.1097/00004583-200212000-00016

Mandell, D. S., Wiggins, L. D., Carpenter, L. A., Daniels, J., DiGuiseppi, C., Durkin, M. S., Giarelli, E., Morrier, M. J., Nicholas, J. S., Pinto-Martin, J. A., Shattuck, P. T., Thomas, K. C., Yeargin-Allsopp, M., \& Kirby, R. S. (2009). Racial/Ethnic Disparities in the Identification of Children with Autism Spectrum Disorders. American Journal of Public Health, 99, 493-498. https://doi.org/10.2105/AJPH.2007.131243

Matson, J. L. (2007). Current Status of Differential Diagnosis for Children with Autism Spectrum Disorders. Research in Developmental Disabilities, 28, 109-118. https://doi.org/10.1016/j.ridd.2005.07.005

Matson, J. L., \& Minshawi, N. F. (2006). Early Intervention for Autism Spectrum Disorders. A Critical Analysis. Oxford, England: Elsevier Science, Inc.

McCulloch, C. E. (2000). Generalized Linear Models. Journal of the American Statistical Association, 95, 452, 1320-1324. https://doi.org/10.1080/01621459.2000.10474340

Mundy, P., \& Burnette, C. (2005). Joint Attention and Neurodevelopmental Models of Autism. In F. R. Volkmar, R. Paul, A. Klin, \& D. Cohen (Eds.), Handbook of Autism and Pervasive Developmental Disorders: Diagnosis, Development, Neurobiology, and Behavior (pp. 650-681). John Wiley \& Sons Inc. https://doi.org/10.1002/9780470939345.ch25

Osterling, I. J., Swinkels, S. H., Jan can der Gaag, R., Visser, J. C., Dietz, C., \& Buitelaar, J. K. (2009). Comparative Analysis of Three Screening Instruments for Autism Spectrum Disorders in Toddlers at High Risk. Journal of Autism and Developmental Disorders, 39, 897-909. https://doi.org/10.1007/s10803-009-0692-9

Rutter, M., Le Couteur, A., \& Lord, C. (2003). ADI-R: Autism Diagnostic Interview-Revised $(A D I-R)$.

Schopler, E., Reichler, R. J., \& Renner, B. (1986). The Childhood Autism Rating Scale (CARS) for Diagnostic Screening and Classification of Autism. New York: Irvington.

Ventola, P., Kleinman, J., Pandey, J. et al. (2007). Differentiating between Autism Spectrum Disorders and Other Developmental Disabilities in Children Who Failed a Screening Instrument for ASD. Journal of Autism and Developmental Disorders, 37, 425-436. https://doi.org/10.1007/s10803-006-0177-z

Volkmar, F., Chawarska, K., \& Klin, A. (2005). Autism in Infancy and Early Childhood. Annual Review of Psychology, 56, 315-336. https://doi.org/10.1146/annurev.psych.56.091103.070159

Wiggins, L. D., \& Robins, D. L. (2008). Brief Report: Excluding the ADI-R Behavioral Domain Improves Diagnostic Agreement in Toddlers. Journal of Autism and Developmental Disorders, 38, 972-976. https://doi.org/10.1007/s10803-007-0456-3

Zwaigenbaum, L., Bauman, M. L., Fein, D., Pierce, K., Buie, T., Davis, P. A. et al. (2015). Early Screening of Autism Spectrum Disorder: Recommendations for Practice and Research. Pediatrics, 136, S41-S59. https://doi.org/10.1542/peds.2014-3667D 